\title{
Beyond Impedance Spectroscopy of Perovskite Solar Cells: Insights from the Spectral Correlation of the Electrooptical Frequency Techniques
}

Agustín Bou, Adam Pockett,* Dimitrios Raptis, Trystan Watson, Matthew J. Carnie, and Juan Bisquert*

Cite This: J. Phys. Chem. Lett. 2020, 11, 8654-8659

Read Online

ACCESS | Lلll Metrics \& More | 国 Article Recommendations | st Supporting Information

ABSTRACT: Small perturbation techniques have proven to be useful tools for the investigation of perovskite solar cells. A correct interpretation of the spectra given by impedance spectroscopy (IS), intensity-modulated photocurrent spectroscopy (IMPS), and intensity-modulated photovoltage spectroscopy (IMVS) is key for the understanding of device operation. The utilization of a correct equivalent circuit to extract real parameters is essential to make this good interpretation. In this work, we present an equivalent circuit, which is able to reproduce the general and the exotic behaviors found in impedance spectra. From the measurements, we demonstrate that the midfrequency features that may appear to depend on the active layer thickness, and we also prove the spectral correlation of the three techniques that has been suggested theoretically.

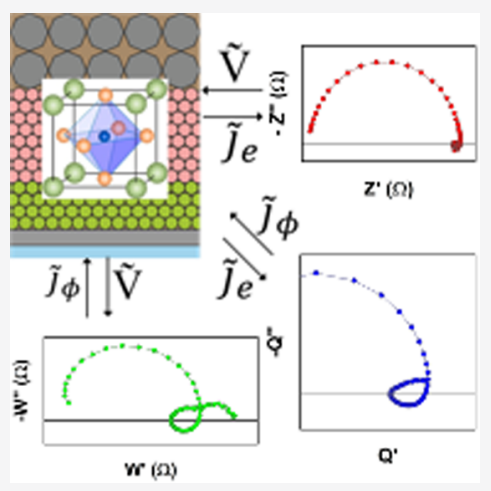

$\mathrm{P}$ erovskite solar cells' record efficiencies have rapidly risen over the past decade thanks to advances and progress in device design and engineering. The improvements in the active layer, the selective contacts, and their interfaces have made perovskite solar cells a real candidate for commercialization. Understanding the electronic and ionic processes taking place in the bulk and contact layers is key for the control of the device quality and for the development of this technology.

Impedance spectroscopy (IS) is a useful technique to characterize physical processes in solar cell devices, and it has been applied extensively in perovskite solar cell research. However, the interpretation and analysis of IS results requires the use of a suitable equivalent circuit (EC). The search for an appropriate EC has been an aim among the perovskite community in the past years, ${ }^{1-5}$ given the fact that a suitable EC would allow the extraction of important parameters of the operation of perovskite solar cells. The wide variety of different spectra reported in the literature makes the convergence to a unique EC a complicated objective.

The majority of perovskite IS complex plots show two distinguishable arcs. However, the combination of both IS and intensity-modulated photocurrent spectroscopy (IMPS) has led to a better distinction of three different features. ${ }^{6}$ This work introduced a novelty in the EC to explain the midfrequency arcs. In addition, the combination of time transient and capacitive methods shows an evolution of capacitance related to ionic dynamics. ${ }^{7,8}$ Another important feature to take into account in IS spectra is the negative capacitance. ${ }^{9-12}$ This feature has been reported in a variety of publications, and its meaning is still under debate. ${ }^{5,13-15}$ It usually appears in the lowest-frequency region of the spectra, and it shows up under different conditions, which makes it more complex to interpret. Different ECs have been proposed for the reproduction of experimental data. ${ }^{5,9,10,12,13,16} \mathrm{~A}$ negative capacitance could have a response due to distinct classes of phenomena. On the one hand, it is the interaction of ionic and electronic effects that conspires to produce delayed dynamics. On the other hand, it is a possible intrinsic instability of the device during the time of measurement of IS. This last phenomenon is clearly observed in perovskite memristors. ${ }^{17}$

The aim of this paper is to describe joint experimental measurements of IS, IMPS, and intensity-modulated photovoltage spectroscopy (IMVS) in carbon-based perovskite solar cells with IS spectra that include negative capacitance features under open circuit conditions. Based on the observations, we wish to point out the distinct spectral features that are obtained in the combination of methods. Due to the lack of previous systematic studies, the election of a proper EC is not an easy question. Here, we propose a new EC that is able to reproduce spectra with midfrequency arcs and negative capacitances, based on previous studies. ${ }^{6,13}$ Earlier studies have also shown

Received: August 12, 2020

Accepted: September 21, 2020

Published: September 21, 2020 
is

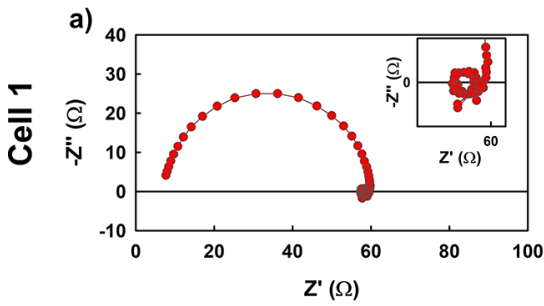

d)
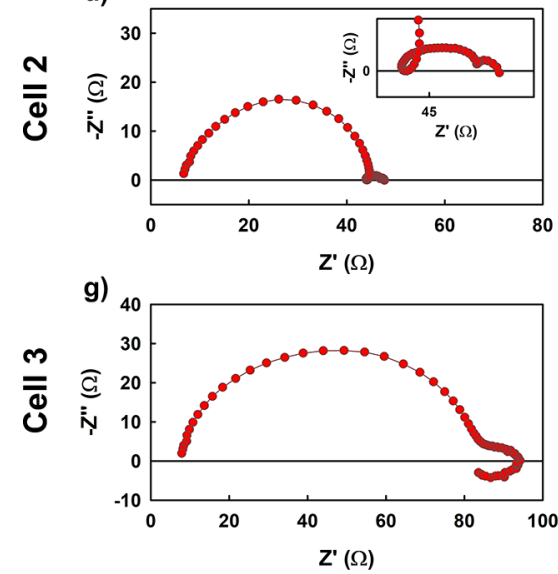

IMPS

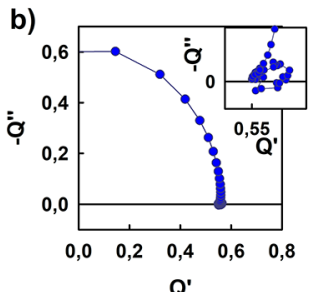

e) 0,4

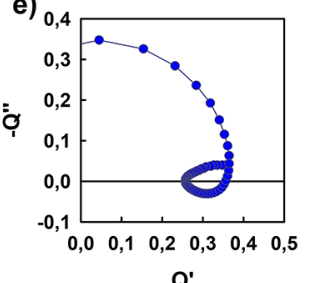

h)

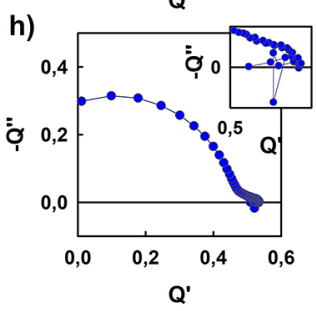

IMVS
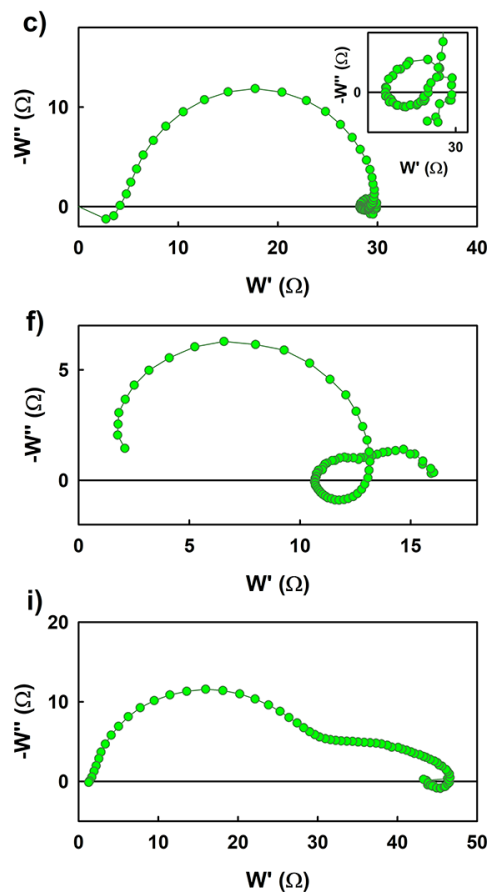

Figure 1. Complex plane plots of the IS (red), IMPS (blue), and IMVS (green) transfer functions $(Z, Q$ and $W$, respectively) for the three devices measured under 0.1 sun of illumination, for a frequency range of $1 \mathrm{MHz}$ to $0.01 \mathrm{~Hz}$ and at open circuit voltage. (a-c) are the spectra for Cell 1 ; $(\mathrm{d}-\mathrm{f})$ are the spectra for Cell 2 ; and $(\mathrm{g}-\mathrm{i})$ are the spectra for Cell 3.

results of the use of the three techniques together for perovskite solar cells. ${ }^{18}$ Here, we aim to clarify how the occurrence of the negative capacitance will affect the shape of the IMPS and IMVS spectra as a tool for interpretation of experiments.

For this work, we chose carbon-based perovskite solar cells, because although they take some time to stabilize, once they are stabilized, they are more stable than other architectures, and therefore, the reproducibility of the measurements is optimal. Moreover, they are a good candidate for addressing issues regarding large scale production. These solar cells consist of a scaffold of mesoporous $\mathrm{TiO}_{2}$ and $\mathrm{ZrO}_{2}$ layers infiltrated with perovskite and do not require a holeconducting layer; see the structure of the cell in Figure S1. ${ }^{19}$ These devices have a thicker perovskite active layer than regular devices, and this could help see interesting spectra. In fact, carbon cells have shown very slow dynamic phenomena in voltage decay methods ${ }^{20,21}$ and tunable hysteresis. ${ }^{22}$ In this work, we used three kinds of devices with the same structure where the only change was the thickness of the zirconia layer and, therefore, the perovskite layer. We have chosen three thicknesses: Cells 1, 2, and 3 correspond to regular, double, and triple thicknesses, respectively. Since the contacts and configuration are the same in all cells, any change in the spectral shapes can be directly related to the perovskite thickness. The current-voltage characteristics of the cells are detailed in Figure S2.

The spectra of IS, IMPS, and IMVS for each cell are shown in Figure 1. If we want to compare all the measurements of the three techniques, it is important that all of them are made under the same conditions. Since IMVS measures the photovoltage change under a small perturbation in the light intensity, it takes place at open circuit conditions. Therefore, all the measurements were made at open circuit voltage.
The IS measurements were performed in the first instance. The fact that the literature about this technique is much more extensive makes it easier to distinguish common features. A representative spectrum of each cell is shown in Figure S2. In general, a big high-frequency arc is common for the three cells, attributed to the geometrical capacitance and the recombination resistance. However, the features that appear next are not as common. We can immediately see inductive loops, negative capacitances, and intermediate frequency arcs. The analysis of the extracted parameters from IS is presented below. As mentioned above, the combination of more than one small perturbation technique has been demonstrated to help distinguish features happening at different frequencies in perovskite solar cells. ${ }^{6}$ After negative capacitance features were detected in every device, it is a great opportunity to study how the presence of the negative capacitance affects the shape of IMPS and IMVS spectra.

In the second instance, we measured IMPS. The complex plane plots of the IMPS transfer function $Q$ are shown in Figure $1(\mathrm{~b}, \mathrm{e}, \mathrm{h})$ (blue). Again, we have a common feature in the high-frequency region: a bigger arc coming from the second quadrant. We find differences in the midfrequency region. Cell 1 shows another small arc with an intermediate loop in the first quadrant. Cell 2 goes into the fourth quadrant forming an intermediate frequency arc there and then coming back to the first quadrant, where it forms a low-frequency arc. Cell 3 forms another arc in the first quadrant, and the lowestfrequency points go slightly into the fourth quadrant, although this measurement is noisy at low frequency, and the points do not form a clear shape. Unlike the previous studies, ${ }^{6,23,24}$ the low-frequency part of the spectra remains or comes back to the first quadrant; this could be linked to the negative capacitance in IS spectra. 
In the third instance, we measured IMVS. This is a technique for which spectra are not usually shown in the literature when talking about perovskite solar cells; instead, they only extract time constants. However, when converting the illumination intensity into current units, the IMVS transfer function $(W)$ takes the same units as the IS transfer function $(Z),{ }^{25}$ so their spectra may be comparable. In fact, we found spectra with similar shapes to those of IS, as we see in Figure $1(c, f, i)$ (green). Although the size of the different features changes somewhat, when comparing $Z$ (red) with $W$ (green), we see a significant correlation regarding the amount and kind of characteristics of the spectra. The change in the size of IMVS features compared to IS has previously been observed for midfrequency characteristics. ${ }^{26}$

Usually, impedance spectra of perovskite solar cells show only two well-separated arcs. The high-frequency arc is related to bulk recombination and the geometrical capacitance, while the low-frequency arc is related to interfacial phenomena. The large capacitance at low frequency has been interpreted as an accumulation of both ionic and electronic charge in the interfaces of the perovskite with the selective contacts. ${ }^{27}$ The most widely used equivalent circuit for impedance spectra data fitting is shown in Figure 2. We will call this circuit Standard

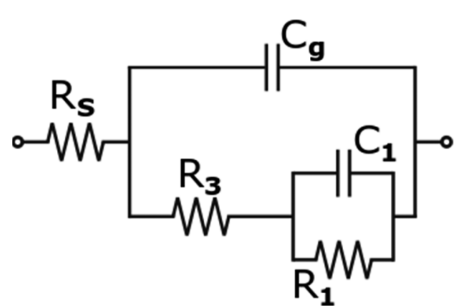

Figure 2. Standard EC used for the analysis of impedance spectra of perovskite solar cells.

EC. Here, the low-frequency resistance and capacitance are $R_{1}$ and $C_{1}$, respectively, and $R_{3}$ is the high-frequency resistance. $C_{\mathrm{g}}$ is the geometrical capacitance, and $R_{\mathrm{S}}$ is the series resistance.

This circuit is only able to produce impedance spectra with one or two arcs in the complex plane plot. However, perovskite spectra showing more features, either at intermediate or at low frequency, are not unusual, as we have seen in Figure S3. In fact, intermediate frequency features that can be hindered in impedance spectra may appear in IMPS spectra of the same cell. This is due to the fact that the time constants of IS features are different from those of IMPS $\left(\tau_{\mathrm{HF}, \mathrm{IMPS}}=R_{\mathrm{S}} C_{\mathrm{g}}\right.$, $\tau_{\mathrm{HF}, \mathrm{IS}}=R_{3} C_{\mathrm{g}}$ ), so that features that appear distinct in one technique become combined in the other one, as previously discussed. ${ }^{6}$ The standard EC is able to produce IMPS spectra with only two $\operatorname{arcs}^{23}$ as shown in Figure S4. Therefore, we can conclude that the Standard EC is too simple for explaining complex spectra with more features, as remarked in the literature. ${ }^{9}$

As remarked above, the observation of negative capacitance is very common, but the physical origin remains uncertain. ${ }^{5,13-15}$ One hypothesis is that the interaction of ionic and electronic effects generates delayed dynamics, either by charge accumulation at the interface ${ }^{13}$ or by ionic redistribution controlling charge injection. ${ }^{28}$ It may also be an intrinsic instability of the device during the time of measurement of IS ${ }^{17}$ or only an apparent effect. ${ }^{14}$ Based on these approaches, we adopt the model proposed by Ghahremanirad et al. for including the negative capacitance features in the EC, ${ }^{13}$ shown in the lower branch of Figure 3a. This method is based on a surface polarization model $^{29}$ that provides a quantitative
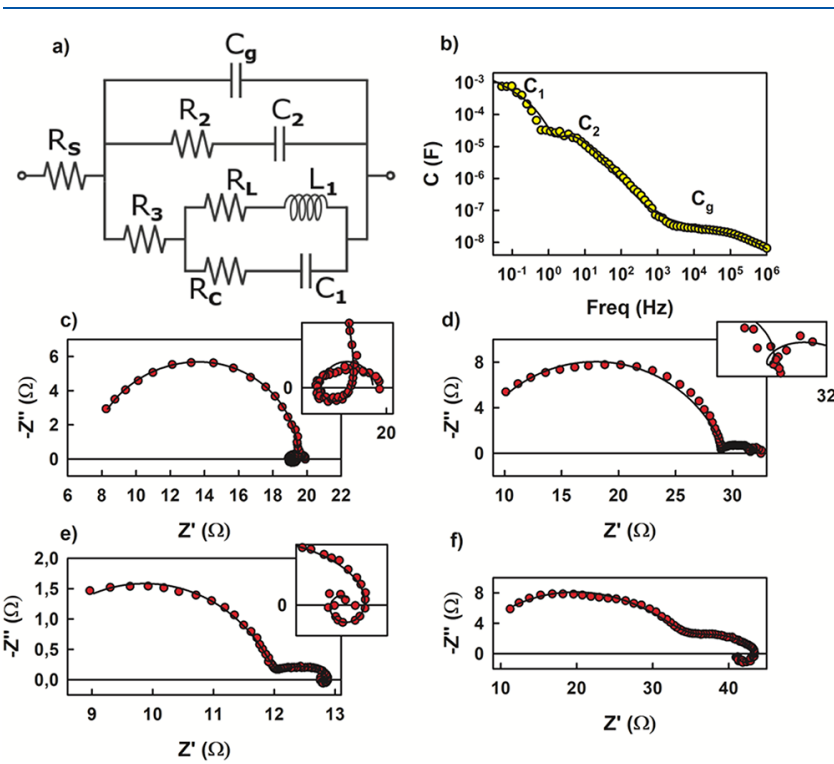

Figure 3. (a) Equivalent circuit for the data fitting of IS of carbonbased perovskite solar cells. (b) Capacitance-frequency plot of Cell 2 showing three capacitances at different frequencies. $(\mathrm{d}-\mathrm{f})$ Impedance complex plots at open circuit conditions. Red and yellow points are experimental data, and the straight black line is the fitting given by the EC. (c) is for Cell 1, (d,e) are for Cell 2, and (f) is for Cell 3.

description of the slow dynamics associated with the ionicelectronic coupling at the interface. The model describes the evolution of an internal surface voltage $V_{\mathrm{s}}$ (related to interfacial polarization) toward equilibrium dictated by the external voltage $V$ and the built-in potential $V_{\mathrm{bi}}$ according to the relaxation equation

$$
\frac{\mathrm{d} V_{\mathrm{s}}}{\mathrm{d} t}=-\frac{V_{\mathrm{s}}-\left(V-V_{\mathrm{bi}}\right)}{\tau_{\text {kin }}}
$$

The EC parameters (including the inductor element) are determined from the physical parameters as described by Ghahremanirad et al. ${ }^{13}$ The time constant $\tau_{\text {kin }}$ has been found in the range of $1-10 \mathrm{~s}$, and it has been linked to ionic binding phenomena at the interface. ${ }^{30}$ This EC is able to fit both negative capacitances at low frequency and inductive loops at midfrequency values, and both features appear in the solar cells that we are presenting. However, this circuit is not able to reproduce midfrequency arcs like the circuit proposed by Ravishankar et al. ${ }^{6}$ for IMPS and IS. Therefore, we propose in Figure $3 \mathrm{a}$ a generalized EC able to reproduce all these features, altogether.

The generalized EC has three parallel branches with three different capacitances that are able to generate three separated features. The elements are ordered according to the frequency in which they appear, i.e., number 1 goes with the lowestfrequency elements, and number 3 goes with the highest; number 2 is for midfrequency, following the established convention. ${ }^{9}$ Figure $3 \mathrm{~b}$ shows a capacitance-frequency $(C-f)$ plot that clearly shows the appearance of three capacitances in the impedance measurements. This $C-f$ plot corresponds to the impedance plot in Figure $3 \mathrm{~d}$. The proper functioning of the circuit is demonstrated in Figure $3 c-f$, where we can see how the same circuit gives a good fitting for a variety of spectra 
showing different phenomena at mid and low frequencies, such as inductive loops, midfrequency arcs, or negative capacitances. Here, the red points are the experimental data measured for different cells, at different illuminations and at open-circuit voltage, and the straight line is the fitting given by the EC in Figure 3a.

By being able to fit all these different spectra, this EC proves to be appropriate for the study of perovskite solar cells. From the fitted spectra, we can extract some conclusions. In Figure $4 \mathrm{a}$ (orange), we can see that the value of $C_{2}$, i.e., the
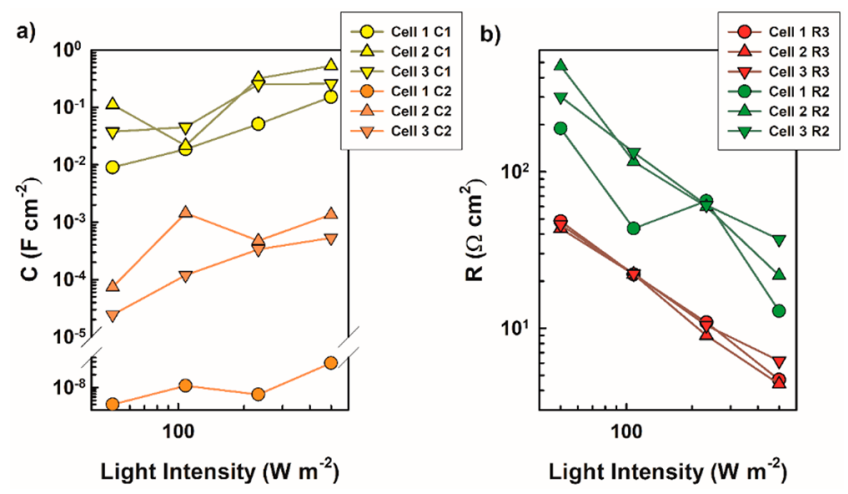

Figure 4. Values of $C_{1}$ and $C_{2}$ (a) and $R_{2}$ and $R_{3}$ (b) depending on the light intensity for the three cells.

midfrequency capacitance, depends on the cell thickness. The values are several orders of magnitude lower for Cell 1 , in comparison with Cell 2 and Cell 3, which have much thicker perovskite layers. In contrast, $C_{1}$ (yellow) takes similar values for all the cells. This means that the intermediate frequency capacitance depends on the perovskite layer thickness, while the low-frequency capacitance does not and can be attributed to surface phenomena. ${ }^{27}$ Moreover, if we look into the values of $R_{3}$ and $R_{2}$ (Figure $4 \mathrm{~b}$ ), we can clearly see that the highfrequency resistance does not depend on the thickness, while the midfrequency resistances are normally higher for Cells 2 and 3. Therefore, the midfrequency elements change with the perovskite thickness.

The resistances and the arcs from IS spectra vary from one cell to another. However, the evolution of all the resistances in one cell with voltage is similar for all the resistances. Therefore, there is a concordance with all the resistances in each cell. This can be seen in Figure S5.

Coming back to Figure 1, when comparing IS and IMVS spectra, the similarities are easily seen. Interestingly, we have found that the IMVS spectra are similar to the IS spectra in the number and the type of characteristics they have, although they are not exactly equal. Moreover, the low-frequency features, which are key for the correct understanding of perovskite solar cell operation, seem to be amplified and more easily distinguishable. Therefore, IMVS can help support the results obtained in IS measurements as well as improve the analysis of those mid- and low-frequency loops and arcs.

The fact that we are doing all measurements under the same conditions, and the three measurement perturbation variables $\left(V, j_{\mathrm{e}}\right.$, and $\left.j_{\mathrm{ph}}\right)$ appear in pairs in the three different techniques, suggests that the three techniques may be linked. This has been previously suggested for dye-sensitized solar cells ${ }^{31}$ and shown with a general derivation by Bertoluzzi et al. ${ }^{25}$ When taken in the same conditions, the three techniques are related by the following equation

$$
Q(\omega)=\frac{W(\omega)}{Z(\omega)}
$$

Therefore, if the measurements are correct, the values for the IMPS transfer function $Q$ should be similar when obtained directly from the measurement and by calculating it from eq 2 .

We have calculated IMPS transfer function $Q$ by dividing IMVS and IS transfer functions, $W$ and $Z$, respectively, for each cell. The comparison between the IMPS experimental transfer function from direct measurements and the IMPS calculated transfer function from $W$ and $Z$ is shown in Figure S6. For all cells, both experimental and calculated spectra have the same number of features with similar shapes. This result indicates the good relation between the techniques, giving truthfulness to the results obtained in these measurements.

In summary, we have measured IS in three similar perovskite solar cells with only one difference: the perovskite layer thickness. From the measurements, we conclude that the effects of the intermediate frequency features are amplified by the perovskite thickness as well as the negative capacitance, which is bigger in the thickest cell. Based on the measurements of IMPS and IMVS in perovskite solar cells that show negative capacitance in IS measurements, we have been able to see how this feature manifests in IMPS and IMVS spectra. In the case of IMPS, we see how the fourth quadrant low-frequency common feature ends up coming back to the first quadrant. Regarding IMVS, the spectra look similar to those of IS. We have built an EC for impedance spectra of perovskite solar cells that is able to reproduce and explain intermediate frequency arcs, inductive loops, and negative capacitances. This EC is based on previous studies that have explained these features separately, and here, we aim to unify this feature in one complete EC. Moreover, it has been able to fit a wide variety of spectra shown by a group of perovskite solar cells. Finally, we have checked the relation between the transfer functions of the three techniques by dividing IMVS and IS transfer functions, obtaining a calculated IMPS transfer function, which is comparable with the measured IMPS spectra.

\section{ASSOCIATED CONTENT}

\section{S) Supporting Information}

The Supporting Information is available free of charge at https://pubs.acs.org/doi/10.1021/acs.jpclett.0c02459.

Experimental details on device preparation, schematic illustration of the devices, and characterization methods. $J-V$ characteristics of devices. Detailed IS measurements. IS, IMPS, and IMVS simulations with ECs. Resistance-voltage plots extracted from IS fittings. IMPS comparison figure (PDF)

\section{AUTHOR INFORMATION}

\section{Corresponding Authors}

Adam Pockett - SPECIFIC, Materials Research Center, College of Engineering, Swansea University, Swansea SA1 8EN, United Kingdom; (1) orcid.org/0000-0002-4747-9560; Email: adam.pockett@swansea.ac.uk

Juan Bisquert - Institute of Advanced Materials (INAM), Universitat Jaume I, 12006 Castello, Spain; ㅈorcid.org/ 0000-0003-4987-4887; Email: bisquert@uji.es 


\section{Authors}

Agustín Bou - Institute of Advanced Materials (INAM), Universitat Jaume I, 12006 Castello, Spain; 이이.org/ 0000-0002-7535-5063

Dimitrios Raptis - SPECIFIC, Materials Research Center, College of Engineering, Swansea University, Swansea SA1 8EN, United Kingdom

Trystan Watson - SPECIFIC, Materials Research Center, College of Engineering, Swansea University, Swansea SA1 8EN, United Kingdom; (1) orcid.org/0000-0002-8015-1436

Matthew J. Carnie - SPECIFIC, Materials Research Center, College of Engineering, Swansea University, Swansea SA1 8EN, United Kingdom; (1) orcid.org/0000-0002-4232-1967

Complete contact information is available at:

https://pubs.acs.org/10.1021/acs.jpclett.0c02459

\section{Notes}

The authors declare no competing financial interest.

\section{ACKNOWLEDGMENTS}

J.B. and A.B. thank the Ministerio de Ciencia e Innovación of Spain for support of this work in the project PID2019107348GB-100. A.B. thanks Ministerio de Ciencia e Innovación of Spain for the FPI Fellowship (BES-2017080351). A.P., D.R., T.W., and M.C. would like to thank the EPSRC (EP/N020863/1, EP/R032750/1, EP/M015254/2, EP/P032591/1), the Welsh European Funding Office (SPARC II), and the Welsh Government's Sêr Solar programme for funding.

\section{REFERENCES}

(1) Bisquert, J.; Bertoluzzi, L.; Mora-Sero, I.; Garcia-Belmonte, G. Theory of Impedance and Capacitance Spectroscopy of Solar Cells with Dielectric Relaxation, Drift-Diffusion Transport, and Recombination. J. Phys. Chem. C 2014, 118, 18983-18991.

(2) Guillén, E.; Ramos, F. J.; Anta, J. A.; Ahmad, S. Elucidating Transport-Recombination Mechanisms in Perovskite Solar Cells by Small-Perturbation Techniques. J. Phys. Chem. C 2014, 118, 2291322922.

(3) Miyano, K.; Yanagida, M.; Tripathi, N.; Shirai, Y. Simple Characterization of Electronic Processes in Perovskite Photovoltaic Cells. Appl. Phys. Lett. 2015, 106, 093903.

(4) Todinova, A.; Contreras-Bernal, L.; Salado, M.; Ahmad, S.; Morillo, N.; Idígoras, J.; Anta, J. A. Towards a Universal Approach for the Analysis of Impedance Spectra of Perovskite Solar Cells: Equivalent Circuits and Empirical Analysis. ChemElectroChem 2017, 4, 2891-2901.

(5) Jacobs, D. A.; Shen, H.; Pfeffer, F.; Peng, J.; White, T. P.; Beck, F. J.; Catchpole, K. R. The Two Faces of Capacitance: New Interpretations for Electrical Impedance Measurements of Perovskite Solar Cells and Their Relation to Hysteresis. J. Appl. Phys. 2018, 124, 225702.

(6) Ravishankar, S.; Aranda, C.; Sanchez, S.; Bisquert, J.; Saliba, M.; Garcia-Belmonte, G. Perovskite Solar Cell Modeling Using Light- and Voltage-Modulated Techniques. J. Phys. Chem. C 2019, 123, 64446449.

(7) Klotz, D.; Tumen-Ulzii, G.; Qin, C.; Matsushima, T.; Adachi, C. Detecting and Identifying Reversible Changes in Perovskite Solar Cells by Electrochemical Impedance Spectroscopy. RSC Adv. 2019, 9, 33436-33445.

(8) Futscher, M. H.; Lee, J. M.; McGovern, L.; Muscarella, L. A.; Wang, T.; Haider, M. I.; Fakharuddin, A.; Schmidt-Mende, L.; Ehrler, B. Quantification of Ion Migration in $\mathrm{CH}_{3} \mathrm{NH}_{3} \mathrm{PbI}_{3}$ Perovskite Solar Cells by Transient Capacitance Measurements. Mater. Horiz. 2019, 6, 1497-1503.
(9) Guerrero, A.; Garcia-Belmonte, G.; Mora-Sero, I.; Bisquert, J.; Kang, Y. S.; Jacobsson, T. J.; Correa-Baena, J.-P.; Hagfeldt, A. Properties of Contact and Bulk Impedances in Hybrid Lead Halide Perovskite Solar Cells Including Inductive Loop Elements. J. Phys. Chem. C 2016, 120, 8023-8032.

(10) Zohar, A.; Kedem, N.; Levine, I.; Zohar, D.; Vilan, A.; Ehre, D.; Hodes, G.; Cahen, D. Impedance Spectroscopic Indication for Solid State Electrochemical Reaction in $\left(\mathrm{CH}_{3} \mathrm{NH}_{3}\right) \mathrm{PbI}_{3}$ Films. J. Phys. Chem. Lett. 2016, 7, 191-197.

(11) Anaya, M.; Zhang, W.; Hames, B. C.; Li, Y.; Fabregat-Santiago, F.; Calvo, M. E.; Snaith, H. J.; Míguez, H.; Mora-Seró, I. Electron Injection and Scaffold Effects in Perovskite Solar Cells. J. Mater. Chem. C 2017, 5, 634-644.

(12) Fabregat-Santiago, F.; Kulbak, M.; Zohar, A.; Vallés-Pelarda, M.; Hodes, G.; Cahen, D.; Mora-Seró, I. Deleterious Effect of Negative Capacitance on the Performance of Halide Perovskite Solar Cells. ACS Energy Lett. 2017, 2, 2007-2013.

(13) Ghahremanirad, E.; Bou, A.; Olyaee, S.; Bisquert, J. Inductive Loop in the Impedance Response of Perovskite Solar Cells Explained by Surface Polarization Model. J. Phys. Chem. Lett. 2017, 8, 14021406.

(14) Ebadi, F.; Taghavinia, N.; Mohammadpour, R.; Hagfeldt, A.; Tress, W. Origin of Apparent Light-Enhanced and Negative Capacitance in Perovskite Solar Cells. Nat. Commun. 2019, 10, 1574.

(15) Klotz, D. Negative Capacitance or Inductive Loop? - A General Assessment of a Common low-Frequency Impedance Feature. Electrochem. Commun. 2019, 98, 58-62.

(16) Taibl, S.; Fafilek, G.; Fleig, J. Impedance Spectra of Fe-Doped $\mathrm{SrTiO}_{3}$ Thin Films Upon Bias Voltage: Inductive Loops as a Trace of Ion Motion. Nanoscale 2016, 8, 13954-13966.

(17) Solanki, A.; Guerrero, A.; Zhang, Q.; Bisquert, J.; Sum, T. C. Interfacial Mechanism for Efficient Resistive Switching in Ruddlesden-Popper Perovskites for Non-Volatile Memories. J. Phys. Chem. Lett. 2020, 11, 463-470.

(18) Pockett, A.; Eperon, G. E.; Peltola, T.; Snaith, H. J.; Walker, A.; Peter, L. M.; Cameron, P. J. Characterization of Planar Lead Halide Perovskite Solar Cells by Impedance Spectroscopy, Open-Circuit Photovoltage Decay, and Intensity-Modulated Photovoltage/Photocurrent Spectroscopy. J. Phys. Chem. C 2015, 119, 3456-3465.

(19) Mei, A.; Li, X.; Liu, L.; Ku, Z.; Liu, T.; Rong, Y.; Xu, M.; Hu, M.; Chen, J.; Yang, Y.; et al. A Hole-Conductor-Free, Fully Printable Mesoscopic Perovskite Solar Cell with High Stability. Science 2014, $345,295-298$.

(20) Pockett, A.; Raptis, D.; Meroni, S. M. P.; Baker, J.; Watson, T.; Carnie, M. Origin of Exceptionally Slow Light Soaking Effect in Mesoporous Carbon Perovskite Solar Cells with AVA Additive. J. Phys. Chem. C 2019, 123, 11414-11421.

(21) Bertoluzzi, L.; Sanchez, R. S.; Liu, L.; Lee, J.-W.; Mas-Marza, E.; Han, H.; Park, N.-G.; Mora-Sero, I.; Bisquert, J. Cooperative Kinetics of Depolarization in $\mathrm{CH}_{3} \mathrm{NH}_{3} \mathrm{PbI}_{3}$ Perovskite Solar Cells. Energy Environ. Sci. 2015, 8, 910-915.

(22) Rong, Y.; Hu, Y.; Ravishankar, S.; Liu, H.; Hou, X.; Sheng, Y.; Mei, A.; Wang, Q.; Li, D.; Xu, M.; et al. Tunable Hysteresis Effect for Perovskite Solar Cells. Energy Environ. Sci. 2017, 10, 2383-2391.

(23) Ravishankar, S.; Riquelme, A.; Sarkar, S. K.; Garcia-Batlle, M.; Garcia-Belmonte, G.; Bisquert, J. Intensity-Modulated Photocurrent Spectroscopy and Its Application to Perovskite Solar Cells. J. Phys. Chem. C 2019, 123, 24995-25014.

(24) Ravishankar, S.; Aranda, C.; Boix, P. P.; Anta, J. A.; Bisquert, J.; Garcia-Belmonte, G. Effects of Frequency Dependence of the External Quantum Efficiency of Perovskite Solar Cells. J. Phys. Chem. Lett. 2018, 9, 3099-3104.

(25) Bertoluzzi, L.; Bisquert, J. Investigating the Consistency of Models for Water Splitting Systems by Light and Voltage Modulated Techniques. J. Phys. Chem. Lett. 2017, 8, 172-180.

(26) Pockett, A.; Eperon, G. E.; Sakai, N.; Snaith, H. J.; Peter, L. M.; Cameron, P. J. Microseconds, Milliseconds and Seconds: Deconvoluting the Dynamic Behaviour of Planar Perovskite Solar Cells. Phys. Chem. Chem. Phys. 2017, 19, 5959-5970. 
(27) Zarazua, I.; Bisquert, J.; Garcia-Belmonte, G. Light-Induced Space-Charge Accumulation Zone as Photovoltaic Mechanism in Perovskite Solar Cells. J. Phys. Chem. Lett. 2016, 7, 525-528.

(28) Moia, D.; Gelmetti, I.; Calado, P.; Fisher, W.; Stringer, M.; Game, O.; Hu, Y.; Docampo, P.; Lidzey, D.; Palomares, E.; et al. Ionic-to-Electronic Current Amplification in Hybrid Perovskite Solar Cells: Ionically Gated Transistor-Interface Circuit Model Explains Hysteresis and Impedance of Mixed Conducting Devices. Energy Environ. Sci. 2019, 12, 1296-1308.

(29) Ravishankar, S.; Almora, O.; Echeverría-Arrondo, C.; Ghahremanirad, E.; Aranda, C.; Guerrero, A.; Fabregat-Santiago, F.; Zaban, A.; Garcia-Belmonte, G.; Bisquert, J. Surface Polarization Model for the Dynamic Hysteresis of Perovskite Solar Cells. J. Phys. Chem. Lett. 2017, 8, 915-921.

(30) Wang, H.; Guerrero, A.; Bou, A.; Al-Mayouf, A. M.; Bisquert, J. Kinetic and Material Properties of Interfaces Governing Slow Response and Long Timescale Phenomena in Perovskite Solar Cells. Energy Environ. Sci. 2019, 12, 2054-2079.

(31) Halme, J. Linking Optical and Electrical Small Amplitude Perturbation Techniques for Dynamic Performance Characterization of Dye Solar Cells. Phys. Chem. Chem. Phys. 2011, 13, 12435-12446. 